\title{
Delayed pneumopericardium after total gastrectomy
}

\section{Abstract}

This report presents a rare complication of gastric surgery. The patient presented to emergency department with shortness of breath two months after his discharge from surgical ward following total gastrectomy and esophago-jejunal anastomosis for gastroesophageal junction adenocarcinoma.
Volume 13 Issue 4 - 2020

Turki Al Garni, Sondos Samargandy,

Abdulrahman Almoghairi

Department of cardiology, Prince Sultan Cardiac Center, Saudi

Arabia

Correspondence: Turki Al Garni,Adult Cardiology

Department, Prince Sultan Cardiac Center PO Box: Riyadh

I I 625, Kingdom of Saudi Arabia, Tel+966-0I I 478300;

Email talgarni@hotmail.com

Received: June 14,2020 | Published: July 10, 2020
Abbreviation: $\mathrm{CT}$, computed tomography; CXR, chest $\mathrm{X}$ Ray; ECHO, Echocardiography; SBP, systolic blood pressure; DBP, diastolic blood pressure

\section{Introduction}

Pneumopericardium, has been described as iatrogenic injury, or due to fistula formation after surgery. ${ }^{1-2}$ However, cancer is an important emerging cause of pneumopericardium. Hirani et al described pneumopericardium in 11 patients with different types of malignant diseases. ${ }^{3}$ Treatment was mainly dependent on the hemodynamic compromise and underlying cause.

\section{Learning objectives}

a. Pneumopericardium is rare complication of gastro-esophageal surgery.

b. Due to the rare nature of such cases, individualized decision making is of paramount importance along with immediate recognition and treatment is very crucial to deliver the best care.

\section{Case report}

58 years old male who is heavy smoker and gastroesophageal junction adenocarcinoma post total gastrectomy and esophago- jejunal anastomosis 2 months back, presented to the emergency department complaining of shortness of breath with moderate mild exertion, referred to cardiology for pericardiocentesis.

\section{Medical history}

We are presenting a case of 58years old male who is heavy smoker and with recent total gastrectomy and esophago- jejunal anastomosis 2 months back, presented to the emergency department complaining of shortness of breath with moderate mild exertion.

On initial examination, the patient was found to have borderline BP (SBP 101, DBP 60), diminished air entry at the bilateral right and left inferior lung fields. The cardiac examination was remarkable for normal S1, S2 and distended jugular venous distension of $10 \mathrm{~cm}$. Chest radiography (CXR) showed an intact esophageal stent along with radiolucent rim surrounding the cardiac silhouette (Figure 1). Echocardiogram (echo) revealed an air gap sign echoic dense space at the anterior and basal part of the heart consistent with the pneumopericardium with no signs of tamponade (Figure 2). Computed tomography (CT) scan chest confirmed the presence of pneumopericardium in anterior pericardium along with left sided mediastinal pocket of air with foci of gas above the esophageal stent likely communicating with the pericardium (Figure 3). Given the clinical and radiological evidence of the symptomatic large pneumopericardium, we consented the patient on an emergency pericardiocentesis. Goals of care were discussed with the patient and his family and the patient opted interventional care measures. A following pericardiocentesis (with off label CT guidance) right sided parasternal approach (marked at the right $5^{\text {th }}$ intercostal space with angulated entry projection of 70 degree medially with $27 \mathrm{~mm}$ depth) was performed successfully with aspiration of $70 \mathrm{ml}$ of air followed with frothy blood-stained aspirate. The gentleman symptoms resolved immediately with picked up BP (SBP 123,DBP 67) and pigtail kept and secured in place for 5 days without any complications. Thereafter, a follow up echo and CT scan repeated and showed bright improvement in the clinical status (Figures $4 \& 5$ ) respectively.

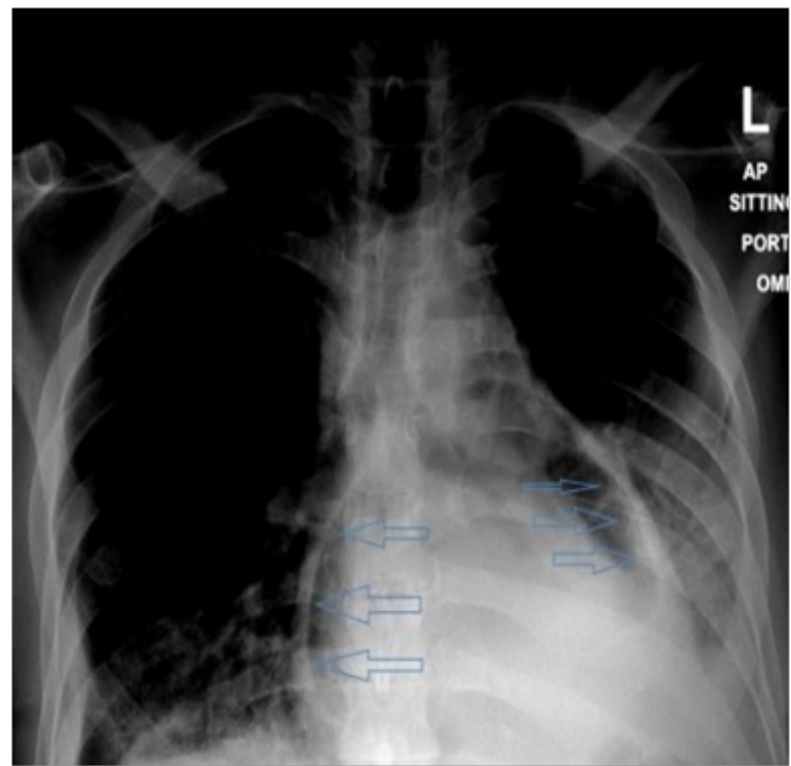

Figure I (Sitting CXR showed radiolucent rim surrounding the cardiac silhouette). 


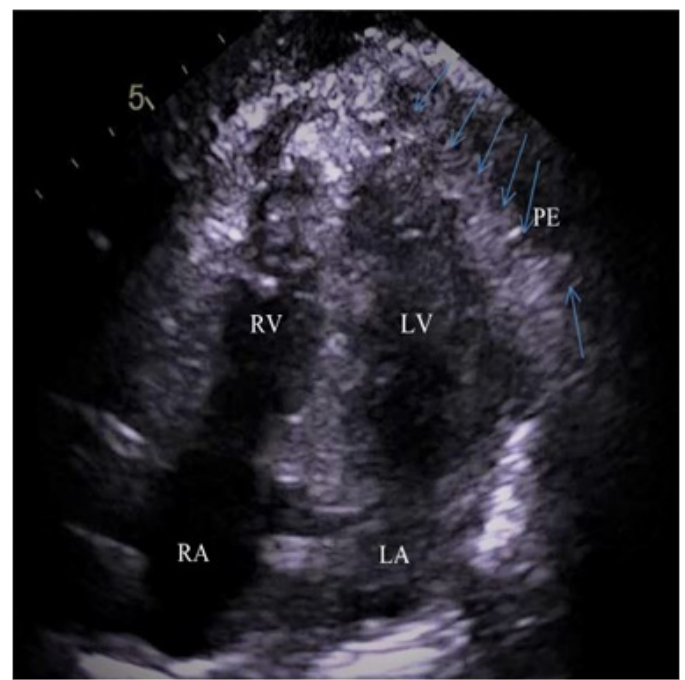

Figure 2 2Dimension -4 Chamber apical view- showed air swirling (blue arrows) along with pericardial effusion (PE) in the pericardial cavity.

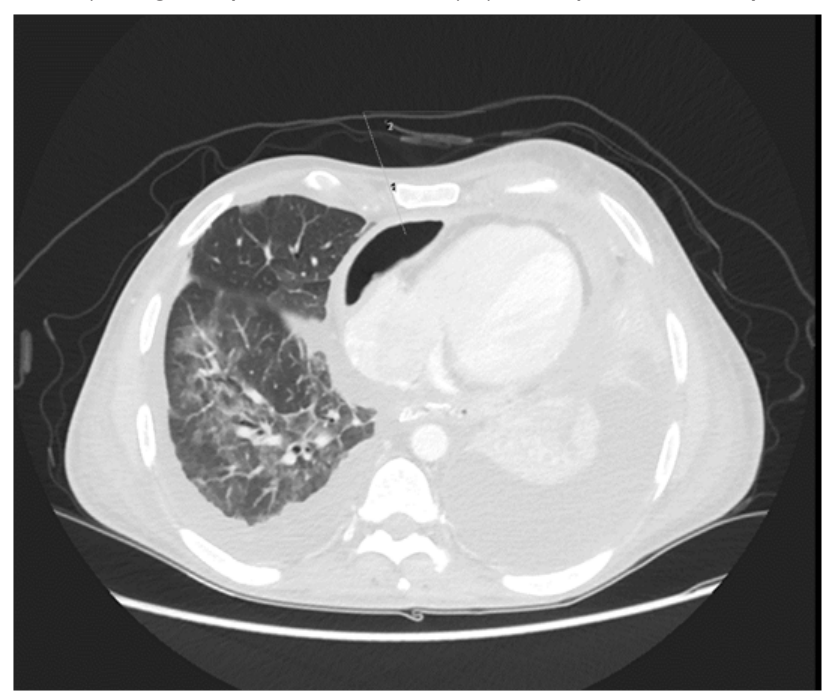

Figure 3 CT axial cut shows anterior Pneumopericardium with proposed entry point at the right 5 th Inter Costal Space with medial inclination of 70 degree and $27 \mathrm{~mm}$ to reach $\mathrm{PE}$.

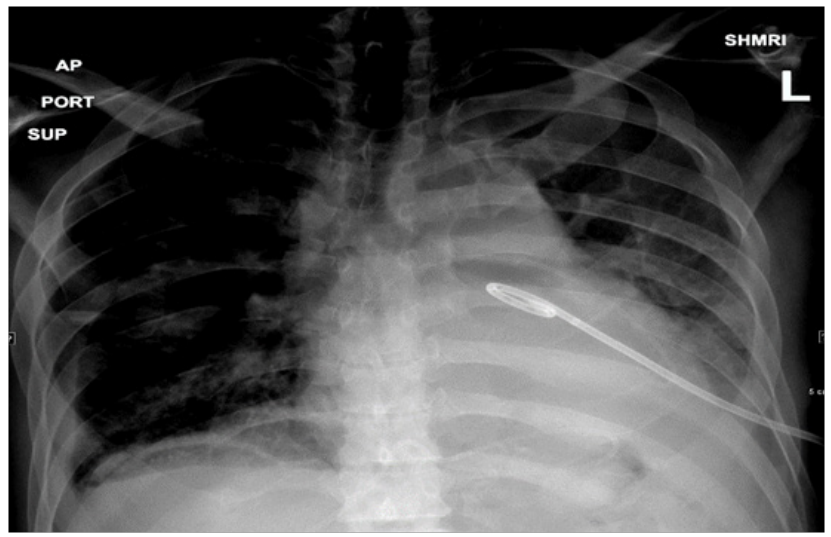

Figure $4 \mathrm{CXR}$ in supine position shows resolution of the radiolucent rim and peg tail in the place around the pericardium.

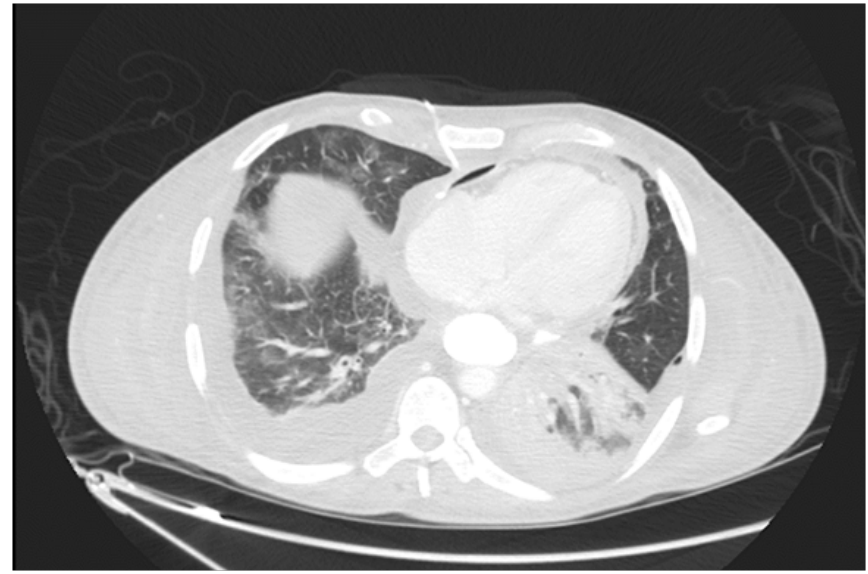

Figure 5 Post Pericardiocentesis CT axial view (LUNG WINDOW) shows pig tail in situ at pericardium with marked improvement.

The samples taken for further work up went to be positive for streptococcus faecium and started on antibiotics as per culture sensitivity report.

\section{Discussion}

With regard to usual clinical presentations, the reported cases were mostly male gender (11 out of 12 including our case) with single female reported 2013 and they attended with dyspnea as most common presenting complain (9 out of 12 cases). ${ }^{3}$ Other frequent symptoms included cough, fever, weight loss, and hemoptysis. Another crucial point to remember is the classical physical exam findings including muffled heart sounds, Haman's crunch and subcutaneous emphysema. ${ }^{4}$ Assessment of hemodynamic stability is very essential cornerstone at the initial assessment. At any point of clinical instability, this should raise a cardiac tamponade flag and require immediate intervention. The diagnosis of pneumopericardium can be obtained with various imaging modalities such as a conventional chest X-ray, CT or echocardiogram. Radiographic findings are usually described as a radiolucent halo that covers the cardiac silhouette. ${ }^{5}$ Based on literature review, a classic signs include small heart signs where a smaller heart is seen on CXR and which consequently return to normal size postintervention, Other common signs included continuous hemidiaphragm signs which are usually seen on the lateral radiograph in which air in the pericardium highlights the normally obscured part of heart. ${ }^{6}$ If the CXR showed an extended air beyond the heart, this means the limits of the pericardium are crossed over to be a more complex situation of pneumomediastinum. Commonly, CXR was followed by a CT scan of the chest for confirmation. It should be noted that because of the anatomical complexities, CT, in particular, is a very valuable informative aid in defining the extent of air, identifying new or recurrent malignancies and the presence of communication between the airway and gastrointestinal system. Identifying the fistula can assist with the management. Echocardiography also serves multiple functions when evaluating pneumopericardium. Most importantly, echocardiography can assess left ventricular compromise as well as facilitating guided pericardiocentesis. ${ }^{7,8}$

Treatment was dependent on the hemodynamic compromise and underlying cause. Gold standard management is pericardiocentesis, usually guided by echocardiography. 


\section{Follow up}

This patient had an eventful hospital course since he had been intubated for sepsis and treated accordingly with good outcome after one week in the intensive care unit. He had been discharged from the hospital symptoms free and without any reported complications to be followed up at the clinic.

\section{Conclusion}

Although pneumopericardium is rare, it should be considered part of the important differential diagnosis screened with rigorous clinical evaluation. The present case highlights the feasibility and safety of CT guided pericardiocentesis. In the current era, the usefulness of CT guided pericardiocentesis giving the synergistic therapeutic and diagnostic (staging) purposes can shed the light on our present case for managing similar cases in the future.

\section{Acknowledgment}

None.

\section{Conflicts of interest}

There were no conflicts of interest during the study.

\section{Funding}

None.

\section{References}

1 van Ede AE, Meis JF, Koot RA, et al. Pneumopericardium complicating invasive pulmonary aspergillosis: case report and review. Infection. 1994;22(2):102-105.

2 Grandhi TM, Rawlings D, Morran CG. Gastropericardial fistula: a case report and review of literature. Emerg Med J. 2004;21(5):644-645.

3 Hirani S, Martinez CSV, Patan S, et al. Cancer-Related Pneumopericardium: A Case Report and Literature Review. Case Rep Oncol. 2020;13(1):23-28.

4 Bejvan SM, Godwin JD. Pneumomediastinum: old signs and new signs. Am J Roentgenol. 1996;166(5):1041-1048.

5 Ghayumi MA, Fallahi MJ, Masoompour SM, et al. Tension hydropneumopericardium after routine intubation. Lancet. 2015;386:e13.

6 Wakabayashi Y, Hayashi T, Mitsuhashi T, et al. Tension pneumopericardium after pericardiocentesis: Useful echocardiographic obscured heart sign and effective postural change during air aspiration. Heart Rhythm. 2018;15(7):1116.

7 Visser F, Heine M, Levin AI, Coetzee AR. Pneumopericardium: two case reports and a review. South Afr J Anaesth Analgesia. 2008;41-45.

8 Russell DA, Boland MJ, Foster JB, et al. Pneumopyopericardium complicating gastric cancer. J Miss State Med Assoc. 1984;25(10):264 266. 